\title{
Gallbladder Hepatoid Adenocarcinoma
}

National Cancer Institute

\section{Source}

National Cancer Institute. Gallbladder Hepatoid Adenocarcinoma. NCI Thesaurus. Code C96890.

A rare variant of gallbladder adenocarcinoma. It is characterized by the presence of a malignant glandular epithelial infiltrate that resembles hepatocellular carcinoma. 